\title{
MAGIC observations of HESS J1809-193 using the Very Large Zenith Angle technique at energies above TeV
}

\author{
Zaric, D., ${ }^{a, *}$ Green, D., ${ }^{b}$ Strzys, M. $^{c}$ and Vovk, I. ${ }^{c}$ on behalf of the MAGIC \\ Collaboration ${ }^{\dagger}$ \\ ${ }^{a}$ University of Split, Faculty of Electrical Engineering, Mechanical Engineering and Naval Architecture \\ (FESB), 21000 Split, Croatia \\ ${ }^{b}$ Max-Planck-Institut für Physik, 80805 München, Germany \\ ${ }^{c}$ Institute for Cosmic Ray Research(ICRR), The University of Tokyo, Kashiwa 277-8582, Chiba, Japan \\ E-mail: Darko.Zaric@fesb.hr
}

The origin of Galactic Cosmic rays (GCRs), whose spectrum extends to PeV energies, is one of the longest-standing problems in astroparticle physics. One of the main sources of GCRs are regarded to be Supernova remnants (SNRs). While SNRs are known to accelerate protons, so far there is no evidence that SNRs can accelerate CRs to PeV energies. Providing that $10 \%$ of the parent Cosmic ray energy is converted to gamma rays, the gamma-ray spectrum extending up to $100 \mathrm{TeV}$ would be a signature of a so-called Galactic PeVatron, an object responsible for the production of protons up to the knee of the Cosmic ray spectrum. The current multi-wavelength data indicate that HESS J1809-193 is one of the most promising Galactic PeVatron candidates. So far, no firm identification on the source nature has been established as there are several possible counterparts at lower energies; one of them being SNR G11.00.0. We report here the results of an observational campaign performed by the MAGIC telescopes on HESS J1809-193 since 2019 in the very-high-energy gamma-ray domain $(\mathrm{E}>100 \mathrm{GeV})$. The data were obtained with the Very Large Zenith Angle (VLZA) technique, which increased the collection area significantly to about one square kilometer. We used 60 hours of collected VLZA data to explore the spectrum and the morphology of the source at energies above several $\mathrm{TeV}$.

$37^{\text {th }}$ International Cosmic Ray Conference (ICRC 2021)

July 12 th - 23rd, 2021

Online - Berlin, Germany

\footnotetext{
${ }^{*}$ Presenter

$\dagger$ a complete list of the MAGIC Collaboration authors can be found at the end of the proceedings
} 


\section{Introduction}

HESS J1809-193 was discovered during the systematic search for very-high-energy (VHE, E $>100 \mathrm{GeV}$ ) $\gamma$-radiation from pulsar wind nebulae in the Galactic plane survey performed by the H.E.S.S. Collaboration ([1]). The $\mathrm{TeV}$ emission of the source has an extension of $0.5^{\circ} \times 0.25^{\circ}$ with most of the emission concentrated in a $0.25^{\circ}$ sub-region. The peak of the VHE emission lies about $6^{\prime}$ to the south of PSR J1809-1917, a middle-aged ( $\left.\tau=51 \mathrm{kyr}\right)$ and energetic $\left(\dot{E}=1.8 \times 10^{36} \mathrm{erg} / \mathrm{s}\right)$ $82.7 \mathrm{~ms}$ radio pulsar. The source spectrum in the $\mathrm{TeV}$ range was fit with a power-law with a spectral index of 2.2. Using radio data from the Karl G. Jansky Very Large Array, a search for the counterpart to the predicted pulsar wind nebula (PWN) generated by PSR J1809-1917 was recently conducted, but no such counterpart was identified. In the analysis by [2], multiple molecular clouds were discovered at the shock front of the SNR G11.0-0.0 with the location of molecular clouds coinciding with the peak of the $\mathrm{TeV}$ gamma-rays. The analysis also revealed evidence for interaction between the SNR and the clouds. According to [2], the distance between the SNR and the clouds is $3 \mathrm{kpc}$. As a result, the authors hypothesized that hadronic interactions can explain the gamma-ray emission.

In the regions of HESS J1809-193, [3] reports the discovery of extended emission in the 0.5-500 $\mathrm{GeV}$ range. The emission has a simple power-law spectrum with a spectral index of $\Gamma \sim 2.2$, which is similar to that of the $\mathrm{TeV}$ source. This is difficult to explain with IC emission from high-energy electrons alone, implying that the emission is hadronic. The hadronic scenario for HESS J1809-193 necessitates a proton spectrum ranging up to $1 \mathrm{PeV}$. However, a deeper measurement of the spectrum at tens of $\mathrm{TeVs}$ is required to confirm its hadronic nature. Other scenarios involving combinations of leptonic and hadronic emission from several known SNRs in the region cannot be ruled out yet.

The notion that HESS J1809-193 is accelerating protons to PeV energy could be important to cosmic ray origin theories. Although the current paradigm for cosmic ray origins implies that SNRs operate as PeVatrons for at least part of their evolution, no known SNR has a gamma-ray spectra that supports this theory. The confirmation of cosmic ray acceleration to PeV energies in the HESS J1809-193/G11.0-0.0 system would be a significant step forward in our knowledge of cosmic ray origins.

\section{Very Large Zenith Observations}

The detection of astrophysical sources at multi-TeV energies may shed light on the mechanism of particle acceleration at the highest energies. In case of Imaging Air Cherenkov Telescopes (IACTs) this requires very long observation times or very large detector arrays, like the upcoming Cherenkov Telescope Array (CTA). The MAGIC Collaboration developed a method for observations at Very Large Zenith Angles (VLZA) [4] by observing above $\sim 60^{\circ}$ zenith, thereby increasing the collections area to $\sim \mathrm{km}^{2}$. Observing in VLZA regime rises the energy threshold to several $\mathrm{TeV}$, which limits the telescope sensitivity to the highest energies.

\section{MAGIC Observations and Datataking}

The MAGIC (Major Atmospheric Gamma Imaging Cherekov) telescopes are two 17m diameter IACTs. They are located on the Canary Island of La Palma (Spain) at the Roque de los Muchachos 
Observatory at $2200 \mathrm{~m}$ above sea level. The telescopes record images of extensive air showers (EAS) in stereoscopic mode and perform observations of VHE gamma-ray sources [5].

The data sample used in this analysis had been accumulated since 2019 in the zenith angle range $50^{\circ}-80^{\circ}$ totalling $\simeq 60 \mathrm{hr}$ of good-quality data taken during dark time after the initial data cleaning. The observations were performed in wobble-mode [6] at symmetrical positions away from the central sky position, which allowed a simultaneous background estimation. The data was taken with wobble offsets of $0.4^{\circ}$ and $0.6^{\circ}$. Diffuse Monte Carlo (MC) data was used to simulate the telescope performance. The MCs had been generated in a way that the events were randomly distributed between 0 and $2.5^{\circ}$ from the camera center, thus simulating an extended source.

\section{Data Analysis}

The collected data was analyzed with standard MAGIC Analysis and Reconstruction Software (MARS) [7]. Events taken during bad weather conditions were discarded. In this case, data taken during median of the aerosol transparency measurements relative to that of an optimal night lower than 0.8 was discarded [8]. In addition to that, a cut on the number of stars visible in the field of view (FoV) of the auxiliary pointing cameras was applied. Standard MARS routines were used to reconstruct the incoming direction, energy and to classify the initiating particles of the extended air showers.

For extended source analysis, a spatial likelihood analysis tool for MAGIC telescope data called SkyPrism [9] was used. The SkyPrism analysis package enables a proper reconstruction of extended sources fluxes over the entire FoV, as the accuracy of the SkyPrism analysis does not degrade with the off-set from the telescope camera centre. SkyPrism uses a 2D spatial modelling (3D if energy is considered) of the measured sky signal. Applying the instrument response to an assumed source model, it can generate an image of the model as it would be seen by the telescope. This model, along with the estimated background map, is fitted to the measured sky image to estimate the most likely flux of the model sources in the chosen sky region.

The chosen method to reconstruct a background exposure model was the so called "Exclusion Map". Using this approach the source itself was excluded in the construction of the background map. Typical selection cuts with $80 \%$ efficiency, based on the MCs, for $\gamma$-ray/hadron separation were used. After the applied analysis cuts the resulting energy threshold of the VLZA dataset was found to be $\sim 2.5 \mathrm{TeV}$. We performed a test-statistic (TS) scan in order to find the source best fit position and extension. The spatial models used were: pointlike, symmetric 2D Gaussian and a disk. As for the spectrum, we tested the power-law, power-law cutoff and log-parabola source models. Background was chosen to be isotropic with a log parabola cutoff spectrum.

\section{Results}

Figure 1 shows the relative flux map as seen by MAGIC compared to recent HAWC measurements [10]. eHWC J1809-193 has similar position and flux to that of the measured MAGIC results. eHWC J1809-193 has measured extension of $\mathrm{R}_{68}=0.51^{\circ} \pm 0.19^{\circ}$ and position within the uncertainty of MAGIC extension of $\mathrm{R}_{68}=0.23^{\circ} \pm 0.07^{\circ}$. A maximum likelihood scan was performed to obtain the source location and extension with Point Source, Disk and 2D symmetric 


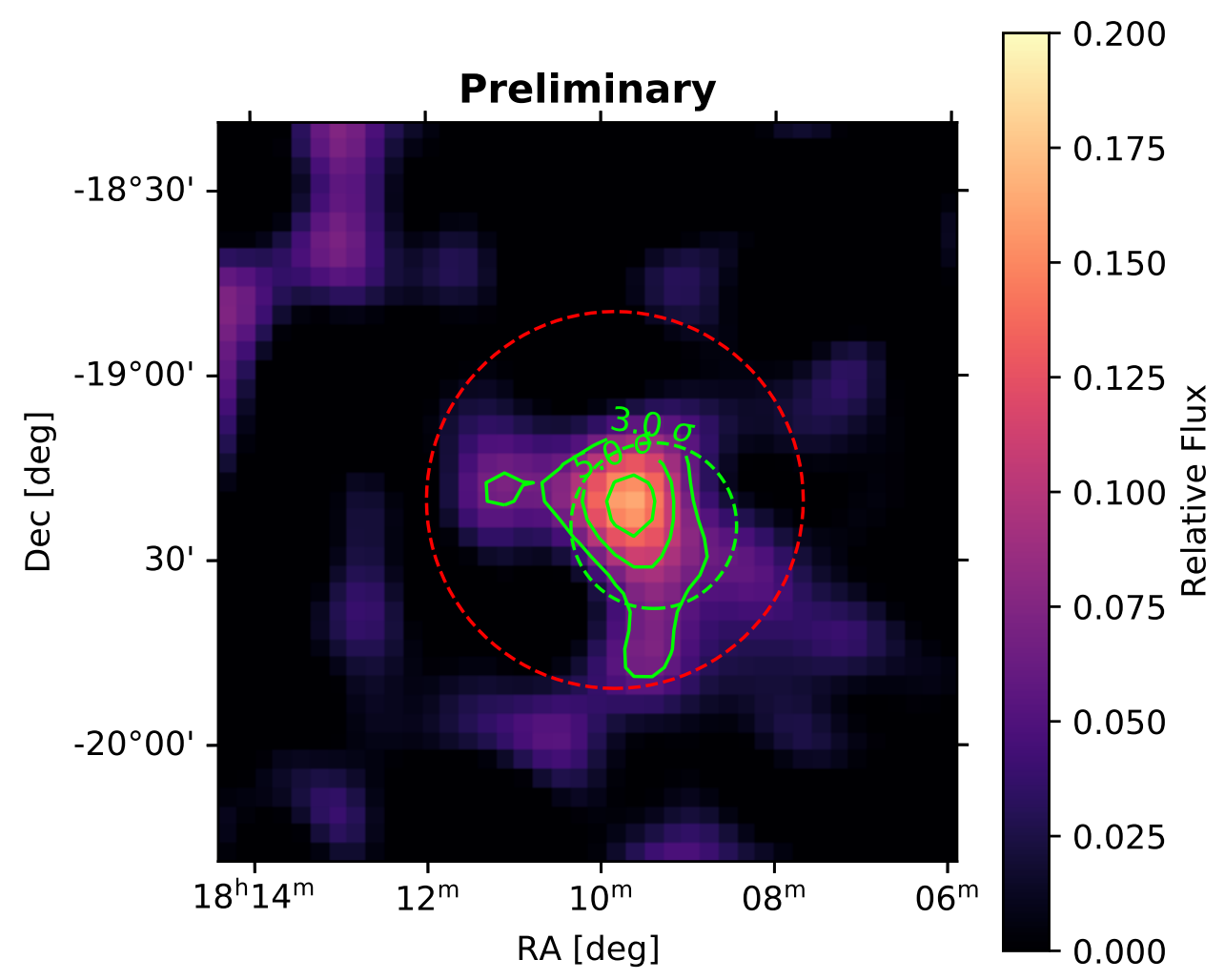

Figure 1: The relative flux skymap of HESS J1809-193 as seen by MAGIC. The skymap covers $3 \mathrm{TeV}$ to $400 \mathrm{TeV}$ in estimated energy. The green dotted circle represents the $\mathrm{R}_{68}$ from the morphology study. The green solid lines represent the $3 \sigma, 5 \sigma$, and $7 \sigma$ contours. The red dashed line is the $\mathrm{R}_{68}$ of eHWC J1809-193 [10]
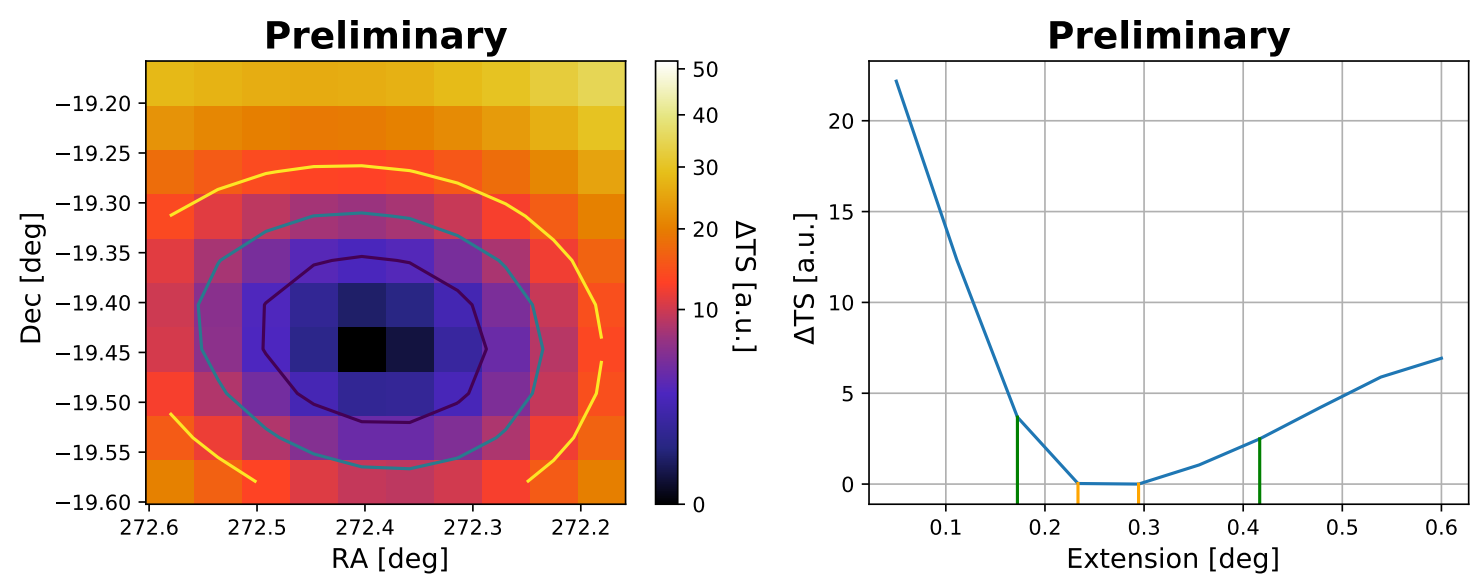

Figure 2: The plots display the results of the TS map scan for the best value for the Ra, Dec and extension using the MAGIC data based on the radially symmetric Gaussian source model. The contours for the 2D plot represent the $1 \sigma$ (purple), $2 \sigma$ (blue), $3 \sigma$ (yellow) errors from this this scan, while for the 1D plots the vertical lines represent the $1 \sigma$ (yellow) and $2 \sigma$ (green) errors from this scan. 
Gaussian as input source models used in the scan. A likelihood ratio test was performed between the point source and the extended models and the 2D symmetric Gaussian spatial model provided the best likelihood value overall. The parameter distribution for the localisation and extension tests of 2D symmetric Gaussian is shown in Figure 2.

For the spectral analysis we used the best spatial model for estimating the spectral parameters. From the forward folding results the Power Law with Exponential Cutoff and Log Parabola were both preferred over a Power Law at about $5 \sigma$ level. The resulting data was then used for the joint forward-folding fit together with Fermi-LAT data and again the Power Law with Exponential Cutoff was preferred over the Power Law. The obtained results are most in line with the recent HAWC measurements [10], which is expected considering the similar extraction region and energy range.

\section{Acknowledgements}

We acknowledge the support from the agencies and organizations listed here:

https://magic.mpp.mpg.de/acknowledgments_ICRC2021/

\section{References}

[1] Aharonian, F., Akhperjanian, A. G., Bazer-Bachi, A. R., Behera, B., Beilicke, M., Benbow, W. et al., Discovery of two candidate pulsar wind nebulae in very-high-energy gamma rays, A\&A 472 (2007) 489.

[2] Castelletti, G., Giacani, E. and Petriella, A., Unveiling the origin of HESS J1809-193, A\&A 587 (2016) A71.

[3] M. Araya, GeV emission in the region of HESS J1809-193 and HESS J1813-178: Is HESS J1809-193 a Proton Pevatron?, The Astrophysical Journal 859 (2018) 69.

[4] R. Mirzoyan, I. Vovk, M. Peresano, P. Temnikov, D. Zaric, N. Godinovic et al., Extending the observation limits of imaging air cherenkov telescopes toward horizon, Nuclear Instruments and Methods in Physics Research Section A: Accelerators, Spectrometers, Detectors and Associated Equipment 952 (2020) 161587.

[5] J. Aleksić et al., The major upgrade of the MAGIC telescopes, Part I: The hardware improvements and the commissioning of the system, Astropart. Phys. 72 (2016) 61 [1409.6073].

[6] V. Fomin, A. Stepanian, R. Lamb, D. Lewis, M. Punch and T. Weekes, New methods of atmospheric Cherenkov imaging for gamma-ray astronomy. I. The false source method, Astroparticle Physics 2 (1994) 137.

[7] MAGIC collaboration, MARS, the MAGIC analysis and reconstruction software, in Proceedings of the 33rd International Cosmic Ray Conference (ICRC2013): Rio de Janeiro, Brazil, July 2-9, 2013, p. 0773, 2013, http://inspirehep.net/record/1412925/files/icrc2013-0773.pdf. 
[8] MAGIC collaboration, A novel LIDAR-based Atmospheric Calibration Method for Improving the Data Analysis of MAGIC, in 33rd International Cosmic Ray Conference, 3, 2014 [1403.3591].

[9] Vovk, I., Strzys, M. and Fruck, C., Spatial likelihood analysis for MAGIC telescope data From instrument response modelling to spectral extraction, A\&A 619 (2018) A7.

[10] HAWC Collaboration collaboration, Multiple Galactic Sources with Emission Above 56 TeV Detected by HAWC, Phys. Rev. Lett. 124 (2020) 021102.

\section{The MAGIC Collaboration}

V. A. Acciari ${ }^{1}$, S. Ansoldi ${ }^{2}{ }^{41}$, L. A. Antonelli ${ }^{3}$, A. Arbet Engels ${ }^{4}$, M. Artero ${ }^{5}$, K. Asano $^{6}$, D. Baack ${ }^{7}$, A. Babic ${ }^{8}$, A. Baquero ${ }^{9}$, U. Barres de Almeida ${ }^{10}$, J. A. Barrio ${ }^{9}$, I. Batković ${ }^{11}$, J. Becerra González ${ }^{1}$, W. Bednarek ${ }^{12}$, L. Bellizzi ${ }^{13}$, E. Bernardini ${ }^{14}$, M. Bernardos ${ }^{11}$, A. Berti ${ }^{15}$, J. Besenrieder ${ }^{15}$, W. Bhattacharyya ${ }^{14}$, C. Bigongiari ${ }^{3}$, A. Biland ${ }^{4}$, O. Blanch ${ }^{5}$, H. Bökenkamp ${ }^{7}$, G. Bonnoli ${ }^{16}$, Ž. Bošnjak ${ }^{8}$, G. Busetto ${ }^{11}$, R. Carosi ${ }^{17}$, G. Ceribella ${ }^{15}$, M. Cerruti ${ }^{18}$, Y. Chai ${ }^{15}$, A. Chilingarian ${ }^{19}$, S. Cikota ${ }^{8}$, S. M. Colak ${ }^{5}$, E. Colombo ${ }^{1}$, J. L. Contreras ${ }^{9}$, J. Cortina ${ }^{20}$, S. Covino ${ }^{3}$, G. D’Amico ${ }^{15,42}$, V. D’Elia ${ }^{3}$, P. Da Vela ${ }^{17,43}$, F. Dazzi ${ }^{3}$, A. De Angelis ${ }^{11}$, B. De Lotto ${ }^{2}$, M. Delfino ${ }^{5,44}$, J. Delgado ${ }^{5,44}$, C. Delgado $M_{e n d e z}^{20}$, D. Depaoli ${ }^{21}$, F. Di Pierro ${ }^{21}$, L. Di Venere ${ }^{22}$, E. Do Souto Espiñeira ${ }^{5}$, D. Dominis Prester $^{23}$, A. Donini ${ }^{2}$, D. Dorner ${ }^{24}$, M. Doro ${ }^{11}$, D. Elsaesser ${ }^{7}$, V. Fallah Ramazani ${ }^{25,45}$, A. Fattorini ${ }^{7}$, M. V. Fonseca ${ }^{9}$, L. Font ${ }^{26}$, C. Fruck ${ }^{15}$, S. Fukami ${ }^{6}$, Y. Fukazawa ${ }^{27}$, R. J. García López ${ }^{1}$, M. Garczarczyk ${ }^{14}$, S. Gasparyan ${ }^{28}$, M. Gaug ${ }^{26}$, N. Giglietto ${ }^{22}$, F. Giordano ${ }^{22}$, P. Gliwny ${ }^{12}$, N. Godinović ${ }^{29}$, J. G. Green ${ }^{3}$, D. Green ${ }^{15}$, D. Hadasch ${ }^{6}$, A. Hahn ${ }^{15}$, L. Heckmann ${ }^{15}$, J. Herrera ${ }^{1}$, J. Hoang ${ }^{9,46}$, D. Hrupec ${ }^{30}$, M. Hütten ${ }^{15}$, T. Inada ${ }^{6}$, K. Ishio ${ }^{12}$, Y. Iwamura ${ }^{6}$, I. Jiménez Martínez ${ }^{20}$, J. Jormanainen ${ }^{25}$, L. Jouvin ${ }^{5}$, M. Karjalainen ${ }^{1}$, D. Kerszberg ${ }^{5}$, Y. Kobayashi ${ }^{6}$, H. Kubo ${ }^{31}$, J. Kushida ${ }^{32}$, A. Lamastra ${ }^{3}$, D. Lelas ${ }^{29}$, F. Leone ${ }^{3}$, E. Lindfors ${ }^{25}$, L. Linhoff ${ }^{7}$, S. Lombardi ${ }^{3}$, F. Longo ${ }^{2,47}$, R. López-Coto ${ }^{11}$, M. López-Moya ${ }^{9}$, A. López-Oramas ${ }^{1}$, S. Loporchio ${ }^{22}$, B. Machado de Oliveira Fraga $^{10}$, C. Maggio ${ }^{26}$, P. Majumdar ${ }^{33}$, M. Makariev ${ }^{34}$, M. Mallamaci ${ }^{11}$, G. Maneva ${ }^{34}$, M. Manganaro ${ }^{23}$, K. Mannheim ${ }^{24}$, L. Maraschi ${ }^{3}$, M. Mariotti ${ }^{11}$, M. Martínez ${ }^{5}$, D. Mazin ${ }^{6,15}$, S. Menchiari ${ }^{13}$, S. Mender ${ }^{7}$, S. Mićanović2 ${ }^{23}$, D. Miceli ${ }^{2,49}$, T. Miener ${ }^{9}$, J. M. Miranda ${ }^{13}$, R. Mirzoyan ${ }^{15}$, E. Molina ${ }^{18}$, A. Moralejo ${ }^{5}$, D. Morcuende ${ }^{9}$, V. Moreno ${ }^{26}$, E. Moretti ${ }^{5}$, T. Nakamori ${ }^{35}$, L. Nava ${ }^{3}$,

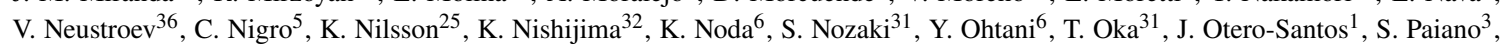
M. Palatiello ${ }^{2}$, D. Paneque ${ }^{15}$, R. Paoletti ${ }^{13}$, J. M. Paredes ${ }^{18}$, L. Pavletić ${ }^{23}$, P. Peñil ${ }^{9}$, M. Persic ${ }^{2,50}$, M. Pihet ${ }^{15}$, P. G. Prada Moroni ${ }^{17}$, E. Prandini ${ }^{11}$, C. Priyadarshi ${ }^{5}$, I. Puljak ${ }^{29}$, W. Rhode ${ }^{7}$, M. Ribó ${ }^{18}$, J. Rico ${ }^{5}$, C. Righi ${ }^{3}$, A. Rugliancich ${ }^{17}$, N. Sahakyan ${ }^{28}$, T. Saito ${ }^{6}$, S. Sakurai ${ }^{6}$, K. Satalecka ${ }^{14}$, F. G. Saturni ${ }^{3}$, B. Schleicher ${ }^{24}$, K. Schmidt ${ }^{7}$, T. Schweizer ${ }^{15}$, J. Sitarek ${ }^{12}$, I. Šnidaric ${ }^{37}$, D. Sobczynska ${ }^{12}$, A. Spolon ${ }^{11}$, A. Stamerra ${ }^{3}$, J. Strišković ${ }^{30}$, D. Strom ${ }^{15}$, M. Strzys ${ }^{6}$, Y. Suda ${ }^{27}$, T. Surić ${ }^{37}$, M. Takahashi ${ }^{6}$, R. Takeishi ${ }^{6}$, F. Tavecchio ${ }^{3}$, P. Temnikov ${ }^{34}$, T. Terzić ${ }^{23}$, M. Teshima ${ }^{15,6}$, L. Tosti ${ }^{38}$, S. Truzzi ${ }^{13}$, A. Tutone ${ }^{3}$, S. Ubach ${ }^{26}$, J. van Scherpenberg ${ }^{15}$, G. Vanzo ${ }^{1}$, M. Vazquez Acosta ${ }^{1}$, S. Ventura ${ }^{13}$, V. Verguilov ${ }^{34}$, C. F. Vigorito ${ }^{21}$, V. Vitale ${ }^{39}$, I. Vovk $^{6}$, M. Will ${ }^{15}$, C. Wunderlich ${ }^{13}$, T. Yamamoto $^{40}$, and D. Zaric ${ }^{29}$

\footnotetext{
${ }^{1}$ Instituto de Astrofísica de Canarias and Dpto. de Astrofísica, Universidad de La Laguna, E-38200, La Laguna, Tenerife, Spain ${ }^{2}$ Università di Udine and INFN Trieste, I-33100 Udine, Italy ${ }^{3}$ National Institute for Astrophysics (INAF), I-00136 Rome, Italy ${ }^{4}$ ETH Zürich, CH-8093 Zürich, Switzerland ${ }^{5}$ Institut de Física d'Altes Energies (IFAE), The Barcelona Institute of Science and Technology (BIST), E-08193 Bellaterra (Barcelona), Spain ${ }^{6}$ Japanese MAGIC Group: Institute for Cosmic Ray Research (ICRR), The University of Tokyo, Kashiwa, 277-8582 Chiba, Japan ${ }^{7}$ Technische Universität Dortmund, D-44221 Dortmund, Germany ${ }^{8}$ Croatian MAGIC Group: University of Zagreb, Faculty of Electrical Engineering and Computing (FER), 10000 Zagreb, Croatia ${ }^{9}$ IPARCOS Institute and EMFTEL Department, Universidad Complutense de Madrid, E-28040 Madrid, Spain ${ }^{10}$ Centro Brasileiro de Pesquisas Físicas (CBPF), 22290-180 URCA, Rio de Janeiro (RJ), Brazil ${ }^{11}$ Università di Padova and INFN, I-35131 Padova, Italy ${ }^{12}$ University of Lodz, Faculty of Physics and Applied Informatics, Department of Astrophysics, 90-236 Lodz, Poland ${ }^{13}$ Università di Siena and INFN Pisa, I-53100 Siena, Italy ${ }^{14}$ Deutsches Elektronen-Synchrotron (DESY), D-15738 Zeuthen, Germany ${ }^{15}$ Max-Planck-Institut für Physik, D-80805 München, Germany ${ }^{16}$ Instituto de Astrofísica de Andalucía-CSIC, Glorieta de la Astronomía s/n, 18008, Granada, Spain ${ }^{17}$ Università di Pisa and INFN Pisa, I-56126 Pisa, Italy ${ }^{18}$ Universitat de Barcelona, ICCUB, IEEC-UB, E-08028 Barcelona, Spain ${ }^{19}$ Armenian MAGIC Group: A. Alikhanyan National Science Laboratory, 0036 Yerevan, Armenia ${ }^{20}$ Centro de Investigaciones Energéticas, Medioambientales y Tecnológicas, E-28040 Madrid, Spain ${ }^{21}$ INFN MAGIC Group: INFN Sezione di Torino and Università degli Studi di Torino, I-10125 Torino, Italy 22 INFN MAGIC Group: INFN Sezione di Bari and Dipartimento Interateneo di Fisica dell'Università e del Politecnico di Bari, I-70125 Bari, Italy ${ }^{23}$ Croatian MAGIC Group: University of Rijeka, Department of Physics, 51000 Rijeka, Croatia ${ }^{24}$ Universität Würzburg, D-97074 Würzburg, Germany ${ }^{25}$ Finnish MAGIC Group: Finnish Centre for Astronomy with ESO, University of Turku, FI-20014 Turku, Finland ${ }^{26}$ Departament de Física, and CERES-IEEC, Universitat Autònoma de Barcelona, E-08193 Bellaterra, Spain 27 Japanese MAGIC Group: Physics Program, Graduate School of Advanced Science and Engineering, Hiroshima University, 739-8526 Hiroshima, Japan ${ }^{28}$ Armenian MAGIC Group: ICRANet-Armenia at NAS RA, 0019 Yerevan, Armenia ${ }^{29}$ Croatian MAGIC Group:
} 
University of Split, Faculty of Electrical Engineering, Mechanical Engineering and Naval Architecture (FESB), 21000 Split, Croatia ${ }^{30}$ Croatian MAGIC Group: Josip Juraj Strossmayer University of Osijek, Department of Physics, 31000 Osijek, Croatia ${ }^{31}$ Japanese MAGIC Group: Department of Physics, Kyoto University, 606-8502 Kyoto, Japan ${ }^{32}$ Japanese MAGIC Group: Department of Physics, Tokai University, Hiratsuka, 259-1292 Kanagawa, Japan ${ }^{33}$ Saha Institute of Nuclear Physics, HBNI, 1/AF Bidhannagar, Salt Lake, Sector-1, Kolkata 700064, India ${ }^{34}$ Inst. for Nucl. Research and Nucl. Energy, Bulgarian Academy of Sciences, BG-1784 Sofia, Bulgaria ${ }^{35}$ Japanese MAGIC Group: Department of Physics, Yamagata University, Yamagata 990-8560, Japan ${ }^{36}$ Finnish MAGIC Group: Astronomy Research Unit, University of Oulu, FI-90014 Oulu, Finland ${ }^{37}$ Croatian MAGIC Group: Ruđer Bošković Institute, 10000 Zagreb, Croatia ${ }^{38}$ INFN MAGIC Group: INFN Sezione di Perugia, I-06123 Perugia, Italy ${ }^{39}$ INFN MAGIC Group: INFN Roma Tor Vergata, I-00133 Roma, Italy ${ }^{40}$ Japanese MAGIC Group: Department of Physics, Konan University, Kobe, Hyogo 6588501, Japan ${ }^{41}$ also at International Center for Relativistic Astrophysics (ICRA), Rome, Italy ${ }^{42}$ now at Department for Physics and Technology, University of Bergen, NO-5020, Norway ${ }^{43}$ now at University of Innsbruck ${ }^{44}$ also at Port d'Informació Científica (PIC), E-08193 Bellaterra (Barcelona), Spain ${ }^{45}$ now at Ruhr-Universität Bochum, Fakultät für Physik und Astronomie, Astronomisches Institut (AIRUB), 44801 Bochum, Germany ${ }^{46}$ now at Department of Astronomy, University of California Berkeley, Berkeley CA 9472047 also at Dipartimento di Fisica, Università di Trieste, I-34127 Trieste, Italy ${ }^{49}$ now at Laboratoire d'Annecy de Physique des Particules (LAPP), CNRS-IN2P3, 74941 Annecy Cedex, France ${ }^{50}$ also at INAF Trieste and Dept. of Physics and Astronomy, University of Bologna, Bologna, Italy 